OPEN ACCESS

Edited by:

Sandra G. Velleman,

Ohio State University at Columbus,

USA

Reviewed by:

Kent M. Reed,

University of Minnesota, USA

Daniel Lee Clark,

Ohio State University at Columbus,

USA

*Correspondence:

Walter G. Bottje

wbottje@uark.edu

Specialty section:

This article was submitted to

Avian Physiology,

a section of the journal

Frontiers in Physiology

Received: 16 March 2017

Accepted: 27 April 2017

Published: 16 May 2017

Citation:

Bottje WG, Lassiter K

Piekarski-Welsher A, Dridi S,

Reverter A, Hudson NJ and Kong B-W (2017) Proteogenomics

Reveals Enriched Ribosome Assembly and Protein Translation in Pectoralis major of High Feed Efficiency Pedigree Broiler Males. Front. Physiol. 8:306. doi: 10.3389/fphys.2017.00306

\section{Proteogenomics Reveals Enriched Ribosome Assembly and Protein Translation in Pectoralis major of High Feed Efficiency Pedigree Broiler Males}

\author{
Walter G. Bottje ${ }^{1 *}$, Kentu Lassiter ${ }^{1}$, Alissa Piekarski-Welsher ${ }^{1}$, Sami Dridi ${ }^{1}$, \\ Antonio Reverter ${ }^{2}$, Nicholas J. Hudson ${ }^{3}$ and Byung-Whi Kong ${ }^{1}$ \\ ${ }^{1}$ Department of Poultry Science, Center of Excellence for Poultry Science, University of Arkansas, Fayetteville, AR, USA, \\ ${ }^{2}$ Computational and Systems Biology, Agriculture and Food (CSIRO), St. Lucia, QLD, Australia, ${ }^{3}$ Animal Science, School of \\ Agriculture and Food Science, University of Queensland, Gatton, QLD, Australia
}

Background: In production animal agriculture, the cost of feed represents $60-70 \%$ of the total cost of raising an animal to market weight. Thus, development of viable biomarkers for feed efficiency (FE, g gain/g feed) to assist in genetic selection of breeding stock remains an important goal in commercial breeding programs.

Methods: Global gene (cDNA microarray, RNAseq) and protein expression (shotgun proteomics) analyses have been conducted on breast muscle samples obtained from pedigree broiler males (PedM) exhibiting high and low FE phenotypes. Using the entire datasets (i.e., no cutoffs for significance or fold difference in expression) the number of genes or proteins that were expressed numerically higher or lower in the high FE compared to the low FE phenotype for key terms or functions, e.g., ribosomal, mitochondrial ribosomal, tRNA, RNA binding motif, RNA polymerase, small nuclear ribonucleoprotein, and protein tyrosine phosphatase, were determined. Bionomial distribution analysis (exact) was then conducted on these datasets to determine significance between numerically up or down expression.

Results: Processes associated with mitochondrial proteome expression (e.g., mitochondrial ribosomal proteins, mitochondrial transcription, mitochondrial tRNA, and translation) were enriched in breast muscle from the high FE compared to the low FE pedigree male broiler phenotype. Furthermore, the high FE phenotype exhibited enrichment of ribosome assembly (e.g., RNA polymerase, mitochondrial and cytosolic ribosomes, small, and heterogeneous nuclear ribonucleoproteins), as well as nuclear transport and protein translation processes compared to the low FE phenotype. Quality control processes (proteosomes and autophagy) were also enriched in the high FE phenotype. In contrast, the low FE phenotype exhibited enrichment of cytoskeletal proteins, protein tyrosine phosphatases, and tyrosine kinases compared to the high FE phenotype. These results suggest that processes of mitochondrial and cytosolic ribosomal construction, activity, and protein translation would be enhanced in high 


\begin{abstract}
FE breast muscle, and that phosphorylation of tyrosine moieties of proteins could be prolonged in the high compared to low FE phenotype. The results indicate the presence of a proteogenomic architecture that could enhance ribosome construction, protein translation, and quality control processes and contribute to the phenotypic expression of feed efficiency in this PedM broiler model.
\end{abstract}

Keywords: feed efficiency, pedigree broiler male, breast muscle, proteomics, transcriptomics, ribosome assembly

\section{INTRODUCTION}

Continued improvement in animal agriculture production efficiency is critical for maintaining sustainable poultry and livestock production. Simultaneously, enhancing feed efficiency (FE) helps meet the protein needs of an ever increasing human population, while making better use of available resources. As animal feed comprises $60-70 \%$ of total cost of raising an animal to market weight across poultry and livestock (see Arthur and Herd, 2005), FE remains an important genetic trait for commercial breeding in poultry and livestock. At the cellular level, the efficiency of how energy is produced and utilized ultimately contributes to cellular utilization efficiency.

In most animal species, skeletal muscle accounts for roughly $50 \%$ of body mass. When considered as a single organ, skeletal muscle contributes between 25 and $40 \%$ of overall basal metabolic rate (Brand, 1990; Zurlo et al., 1990; Rolfe et al., 1999), $25 \%$ of which is attributed to one component of mitochondrial function; i.e., proton leak (Rolfe and Brand, 1996, 1997). A clear link between breast muscle mitochondrial physiology and FE has been reported (Bottje et al., 2002; Bottje and Carstens, 2009). Thus, skeletal muscle mitochondria can play a substantive role in overall efficiency in animals.

In global gene and protein expression studies, emphasis is placed on using cutoff values for fold differences (e.g., $>1.3-$ fold, $P<0.05$ ) between groups which produces well defined, differentially expressed genes or proteins datasets that are verifiable, manageable, and can be discussed in a cohesive manner. This approach enhances understanding of fundamental mechanisms associated with given condition, but a tremendous amount of potentially informative data is discarded from further consideration. This is particularly true for high throughput genomic data where the number of assays (e.g., cDNA probes or RNA reads) vastly exceeds the number of biological replicates. In these cases multiple testing penalties can be very severe and may lead to few, if any, significant differences being identified. Here we present an alternative approach that may especially lend itself to samples with subtle phenotypes such as feed efficiency within a single genetic line. We argue that individually, small effects can be considered significant if they combine cumulatively in a given biological process or pathway.

To obtain more information from global expression datasets, phenotypic and regulatory impact (PIF and RIF) analyses were developed to identify molecules that have a large effect on a given phenotype due to their interconnectedness with many cellular processes, but may not be differentially expressed (e.g., Hudson et al., 2009, 2012). Using RIF analysis, we reported that progesterone signaling plays a role in the phenotypic expression of FE in the skeletal muscle of pedigree male (PedM) broilers (Bottje et al., 2017). It was also reported that the mitochondrial transcriptome and proteome were elevated in the high FE phenotype (based on binomial analysis of numbers of transcripts or proteins that were numerically or significantly higher in high vs. low FE PedM broiler phenotype, Kong B. et al., 2016; Bottje et al., 2017). These studies represent a precedent for using binomial statistics at a particular process level (all mRNA encoding the mitochondrial proteome and all proteins present in the mitochondria) to gather insights that would not have been possible with traditional methods. The common finding by both independent approaches (mRNA and protein) supports the utility of using binomial statistics in this manner.

It is not clear whether the elevation of the mitoproteome we detected in the high FE PedM broilers is a consequence of an elevated mitochondrial content, an elevated mitochondrial activity or some weighted combination of the two. For example, Kong R. S. et al. (2016) recently discovered that more efficient cattle exhibited elevated mitochondrial gene expression but a lower mitochondrial content in rumen epithelia. In order for the mitoproteome to be elevated in the high FE phenotype (Kong B. et al., 2016), we hypothesize that, not only must mitoproteome gene expression be elevated (Bottje et al., 2017), there must also be supportive architecture involving, cytosolic and mitochondrial ribosome expression, RNA synthesis, and specialized ribonucleoproteins such as that provided in a review by Wahl et al. (2009) that would foster mitoproteome expression. Thus, the major goal of this data mining study was to assess the numbers of genes and proteins in global expression datasets in muscle obtained from high and low FE PedM broiler phenotypes associated with upstream components requisite for protein synthesis.

\section{MATERIALS AND METHODS}

\section{Ethics Statement}

The present study was conducted in accordance with the recommendations in the guide for the care and use of laboratory animals of the National Institutes of Health. All procedures for animal care were reviewed and approved by the University of Arkansas Institutional Animal Care and Use Committee (IACUC): Protocol \#14012.

\section{Tissues-Animals}

The global gene and protein expression data examined in this study were obtained previously (Kong et al., 2011; Kong B. et al., 2016; Bottje et al., 2017). The data were all obtained from the same set of breast muscle samples from pedigree male (PedM) 
broilers individually phenotyped for feed efficiency (FE) using procedures previously described (Bottje et al., 2002). Briefly, FE (amount of body weight gain/amount of feed consumed) was determined between 6 and 7 week of age on a group of 100 PedM broilers housed in individual cages in the same environment. All birds received the same corn-soybean based diet $(20.5 \%$ protein, $3,280 \mathrm{kcal} / \mathrm{kg}$ ) and water ad libitum during the 1 week phenotyping period (from 6 to 7 week of age). From this group of 100 birds, those exhibiting the highest and lowest FE ( $n=6$ per group) were obtained. The average weight gain (g), feed intake (FI, g), and FE (gain/feed) for the high and low FE groups is provided in Table 1 . The same tissue samples were analyzed in both the microarray (Kong et al., 2011) and RNAseq (Bottje et al., 2017 ) studies whereas a subset of these samples ( $n=4$ /group) were analyzed in the proteomic study (Kong B. et al., 2016). In Table 1, it is apparent that the high FE PedM broilers achieved better FE by gaining more weight on the same amount of feed consumption compared to the low FE phenotype. After humane killing, breast muscle samples were obtained, flash frozen in liquid nitrogen and stored at $-80^{\circ} \mathrm{C}$ until analysis.

\section{Global Expression Analysis}

Detailed descriptions of global gene expression analysis by cDNA microarray and RNAseq are provided in Kong et al. (2011) and Bottje et al. (2017), respectively. Procedures for global protein expression analysis (shotgun proteomics) are provided in Kong B. et al. (2016). In the cDNA microarray study (Kong et al., 2011), following RNA extraction equal amounts of RNA obtained from 6 separate muscle samples for the high and low FE groups were pooled and hybridized on a $4 \times 44 \mathrm{~K}$ Agilent chicken oligo microarray (array ID: 015068; Agilent Technologies, Inc.) with four replicates of each group in the microarray analysis. An argument for the use of pooled samples in the microarray study was provided in a companion paper (Bottje et al., 2012):

\footnotetext{
"In a comparison of pooled vs. individual sample microarray analysis, Jolly et al. (2005) concluded that 'while the two approaches to running microarray chips were comparable...the individual analysis revealed subtle changes that affect interpretation of the experiment that were lost in the pooled analysis and important for mechanistic understanding. However, justification for use of pooled samples was provided by Kendziorski et al. (2005) who indicated that pooling samples for microarray analysis also minimizes subject-to-subject variation that "is often desirable when primary interest is not on the individual but rather on characteristics of the population from which certain individuals are obtained (e.g., identifying biomarkers or expression patterns common across individuals)." Similarly, in the context of the present paper, we are more interested in looking for gene expression patterns across individuals in the high and low FE phenotypes according to Kendziorski et al. (2005) at the potential expense of losing some mechanistic understanding as indicated by Jolly et al. (2005)."
}

For the RNAseq analysis, RNA was isolated from 6 breast muscle samples per group and analyzed with an Illumina HiSeq using $2 \times 100$ bp paired end read sequencing at the Research Support Facility at Michigan State University (East Lansing, MI) on an Illumina HiSeq using 100 base paired end read
TABLE 1 | Body weight gain (g), feed intake (FI, g), and feed efficiency (FE, gain/FI) of pedigree broiler males (PedM) exhibiting high FE and low FE phenotypes ${ }^{a}$.

\begin{tabular}{|c|c|c|c|c|}
\hline & $n$ & $\begin{array}{l}\text { Body } \\
\text { wt. gain } \\
\text { (g) }\end{array}$ & $\begin{array}{c}\text { Feed } \\
\text { intake }(\mathrm{g})\end{array}$ & $\begin{array}{l}\text { Feed efficiency } \\
\text { (g gain/g feed } \\
\text { intake) }\end{array}$ \\
\hline \multicolumn{5}{|c|}{ MICROARRAY (Kong et al., 2011) and RNAseq (Bottje et al., 2017) } \\
\hline PedM High FE & 6 & $641 \pm 20^{*}$ & $973 \pm 33$ & $0.659 \pm 0.011^{*}$ \\
\hline PedM Low FE & 6 & $485 \pm 17$ & $1048 \pm 34$ & $0.462 \pm 0.006$ \\
\hline \multicolumn{5}{|c|}{ PROTEOMICS (Kong B. et al., 2016) } \\
\hline PedM High FE & 4 & $660 \pm 18^{*}$ & $1019 \pm 21$ & $0.648 \pm 0.007^{*}$ \\
\hline PedM Low FE & 4 & $491 \pm 26$ & $1075 \pm 45$ & $0.456 \pm 0.006$ \\
\hline
\end{tabular}

a Values represent the mean $\pm S E$.

${ }^{b}$ The same muscle tissues ( $n=6$ /group) were analyzed in the microarray and RNAseq studies and a subset of the same muscle tissues ( $n=4 /$ group) were analyzed in the proteomics.

${ }^{*}$ Mean value is greater $(P<0.05)$.

sequencing. Shotgun proteomics analysis was conducted on breast muscle from a total of 4 high and 4 low FE samples by ingel trypsin digestion and tandem mass spectrometry (MS/MS) at the University of Arkansas Medical Science (UAMS) Proteomics Core Lab (Little Rock, AR).

\section{Statistical Analysis}

The number of genes or proteins associated with a specific process were determined by compiling a list of molecules after searching each dataset for specific terms that are provided in Tables 2-5. The numbers of molecules in which mean values were numerically higher $(\mathrm{H})$ or lower $(\mathrm{L})$ in breast muscle of the high FE compared to the low FE PedM phenotype were determined and used in the exact binomial distribution analysis test offered in the 2010 version of Microsoft Excel $^{\mathrm{TM}}$. In this study there was no gating of terms based on significant or fold difference in expression for a given transcript or protein. The mean values for the proteomics dataset (Kong B. et al., 2016) were based on 4 samples per FE phenotype and were a subset obtained from the same 6 samples that were analyzed per FE phenotype in the microarray (Kong et al., 2011) and RNAseq (Bottje et al., 2017) studies.

\section{RESULTS AND DISCUSSION}

\section{Mitochondrial Ribosome Proteins Are Enriched in High FE Breast Muscle}

We reported that mitoproteome expression was enriched in breast muscle of high FE compared to low FE PedM broilers (Kong B. et al., 2016; Bottje et al., 2017). For this to occur, we hypothesize that a genomic architecture must exist that would foster mitochondrial protein synthesis in the high FE phenotype including production of mitochondrial ribosomes (e.g., Graack et al., 1999) and components of RNA synthesis, ribosome assembly, and protein translating activities (e.g., Wahl et al., 2009). A list of all genes associated with the term "mitochondrial ribosomal protein" in the RNAseq dataset (Bottje et al., 2017), is provided in Table 2 as an example of data used in binomial 
TABLE 2 | Mitochondrial ribosomal protein gene expression list obtained from the RNAseq dataset of breast muscle tissue generated by Bottje et al. (2017) showing $\log _{2}$ high feed efficiency- $\log _{2}$ low feed efficiency (M), the gene symbol and the gene name.

\begin{tabular}{|c|c|c|}
\hline M & Symbol & Entrez Gene Name \\
\hline 1.13 & MRPL55 & Mitochondrial ribosomal protein L55 \\
\hline 0.72 & MRPL24 & Mitochondrial ribosomal protein L24 \\
\hline 0.71 & MRPS18C & Mitochondrial ribosomal protein S18C \\
\hline 0.53 & MRPL9 & Mitochondrial ribosomal protein L9 \\
\hline 0.52 & MRPL17 & Mitochondrial ribosomal protein L17 \\
\hline 0.44 & MRPL18 & Mitochondrial ribosomal protein L18 \\
\hline 0.36 & MRPL42 & Mitochondrial ribosomal protein L42 \\
\hline 0.36 & MRPS11 & Mitochondrial ribosomal protein S11 \\
\hline 0.36 & MRPS22 & Mitochondrial ribosomal protein S22 \\
\hline 0.36 & MRPS35 & Mitochondrial ribosomal protein S35 \\
\hline 0.35 & MRPL21 & Mitochondrial ribosomal protein L21 \\
\hline 0.34 & MRPL44 & Mitochondrial ribosomal protein L44 \\
\hline 0.33 & MRPL2 & Mitochondrial ribosomal protein L2 \\
\hline 0.33 & MRPL47 & Mitochondrial ribosomal protein L47 \\
\hline 0.32 & MRPS5 & Mitochondrial ribosomal protein S5 \\
\hline 0.30 & MRPS21 & Mitochondrial ribosomal protein S21 \\
\hline 0.30 & MRPS23 & Mitochondrial ribosomal protein S23 \\
\hline 0.29 & MRPL16 & Mitochondrial ribosomal protein L16 \\
\hline 0.29 & MRPL35 & Mitochondrial ribosomal protein L35 \\
\hline 0.29 & MRPS31 & Mitochondrial ribosomal protein S31 \\
\hline 0.28 & MRPL3 & Mitochondrial ribosomal protein L3 \\
\hline 0.27 & MRPL38 & Mitochondrial ribosomal protein L38 \\
\hline 0.26 & MRPL32 & Mitochondrial ribosomal protein L32 \\
\hline 0.26 & MRPL54 & Mitochondrial ribosomal protein L54 \\
\hline 0.26 & MRPS26 & Mitochondrial ribosomal protein S26 \\
\hline 0.25 & MRPL46 & Mitochondrial ribosomal protein L46 \\
\hline 0.24 & MRPL51 & Mitochondrial ribosomal protein L51 \\
\hline 0.24 & MRPS25 & Mitochondrial ribosomal protein S25 \\
\hline 0.23 & MRPS2 & Mitochondrial ribosomal protein S2 \\
\hline 0.22 & MRPL50 & Mitochondrial ribosomal protein L50 \\
\hline 0.20 & MRPL10 & Mitochondrial ribosomal protein L10 \\
\hline 0.20 & MRPL23 & Mitochondrial ribosomal protein L23 \\
\hline 0.17 & MRPL39 & Mitochondrial ribosomal protein L39 \\
\hline 0.17 & MRPL48 & Mitochondrial ribosomal protein L48 \\
\hline 0.17 & MRPS18A & Mitochondrial ribosomal protein S18A \\
\hline 0.16 & MRPL40 & Mitochondrial ribosomal protein L40 \\
\hline 0.16 & MRPS36 & Mitochondrial ribosomal protein S36 \\
\hline 0.15 & MRPS9 & Mitochondrial ribosomal protein S9 \\
\hline 0.14 & MRPL14 & Mitochondrial ribosomal protein L14 \\
\hline 0.13 & MRPS6 & Mitochondrial ribosomal protein S6 \\
\hline 0.12 & MRPS34 & Mitochondrial ribosomal protein S34 \\
\hline 0.11 & MRPL1 & Mitochondrial ribosomal protein L1 \\
\hline 0.11 & MRPL20 & Mitochondrial ribosomal protein L20 \\
\hline 0.11 & MRPS15 & Mitochondrial ribosomal protein S15 \\
\hline 0.10 & MRPL28 & Mitochondrial ribosomal protein L28 \\
\hline 0.09 & MRPL53 & Mitochondrial ribosomal protein L53 \\
\hline 0.08 & MRPL37 & Mitochondrial ribosomal protein L37 \\
\hline 0.06 & MRPL22 & Mitochondrial ribosomal protein L22 \\
\hline
\end{tabular}

(Continued)
TABLE 2 | Continued

\begin{tabular}{lll}
\hline $\mathbf{M}$ & Symbol & Entrez Gene Name \\
\hline 0.06 & MRPS7 & Mitochondrial ribosomal protein S7 \\
0.05 & MRPS10 & Mitochondrial ribosomal protein S10 \\
0.04 & MRPS14 & Mitochondrial ribosomal protein S14 \\
0.04 & MRPS30 & Mitochondrial ribosomal protein S30 \\
0.02 & MRPL15 & Mitochondrial ribosomal protein L15 \\
0.00 & MRPS27 & Mitochondrial ribosomal protein S27 \\
-0.01 & MRPL41 & Mitochondrial ribosomal protein L41 \\
-0.01 & MRPS33 & Mitochondrial ribosomal protein S33 \\
-0.03 & MRPL13 & Mitochondrial ribosomal protein L13 \\
-0.07 & MRPS17 & Mitochondrial ribosomal protein S17 \\
\hline
\end{tabular}

distribution analysis in Tables 3-5. Instances in which gene and protein expression differences between high and low FE groups were 0 were rare; e.g., only one gene, MRPS27, out of 59 total mitochondrial ribosomal protein transcripts listed in Table $\mathbf{1 .}$ Percentages of genes or proteins that exhibited no difference in mean expression between high and low FE groups in global expression datasets were $0.23 \%$, (4 of 1,806 proteins; Kong B. et al., 2016) and 1.52\% (167 of 10,981 transcripts; Bottje et al., 2017).

Binomial analysis associated with mitochondrial protein expression machinery is provided in Table 3 and depicted in Figure 1. Enrichments favoring the high FE phenotype were observed for genes encoding mitochondrial ribosomal proteins, mitochondrial tRNA, mitochondrial transcription and for inner mitochondrial membrane transport proteins. Mitochondrial ribosomes (55S) are very different from cytoplasmic (80S) and bacterial ribosomes (70S) (Avadhani and Buetow, 1974; O'Brien, 2002). Mitochondrial ribosomes have a greater protein content compared to bacterial and cytoplasmic ribosomes (Sylvester et al., 2004) as well as proteins that are unique to the mitochondrial ribosomes (Graack et al., 1999). In mammals, all mitochondrial ribosomal proteins $(\sim 80)$ are encoded by nuclear genes and translated on cytoplasmic ribosomes prior to import into the mitochondria through transport proteins on the outer (TOMM) and inner (TIMM) mitochondrial membranes (Scheiber and O’Brien, 1985; O’Brien, 2002; Fox, 2012). While there was no difference between FE groups for TOMM, 10 of 11 TIMM transcripts that were detected in the data were higher in the high FE phenotype (Table 2). Once inside, mitochondrial ribosomal proteins assemble into the $39 \mathrm{~S}$ and $28 \mathrm{~S}$ ribosomes that translate messages from mitochondrial (mt) DNA encoded genes that include 13 electron transport chain proteins, the $16 \mathrm{~S}$ and $12 \mathrm{~S}$ rRNA genes and 22 tRNA genes that are essential for synthesis of mitochondrial proteins (Wallace, 1999; Sue and Schon, 2000). Enrichment of mitochondrial transcription, mitochondrial tRNA and for mitochondrial translation and processing of mtDNA encoded proteins was evident in the high FE phenotype (Table 3) and consistent with the enriched mitoproteome in the high FE phenotype (Kong B. et al., 2016; Bottje et al., 2017).

The data presented in Table 3 highlights a well-established short coming of proteomic vs. transcriptomic analyses. 
TABLE 3 | Numbers of genes and proteins associated with mitochondrial protein synthesis (ribosomes, transcription, and tRNA) as well as transport of molecules into the mitochondria that were higher $(\mathrm{H})$ or lower $(\mathrm{L})$ in breast muscle of high feed efficiency (FE) compared to low FE pedigree broiler male for the terms provided obtained from global expression microarray, proteomics, and RNAseq datasets and for combined in all three global expression datasets.

\begin{tabular}{|c|c|c|c|c|c|c|c|c|c|}
\hline Terms & \multicolumn{3}{|c|}{ Microarray ${ }^{a}-$} & \multicolumn{3}{|c|}{ Proteomics $^{b}-$} & \multicolumn{3}{|c|}{ RNAseq $^{\mathrm{C}}-$} \\
\hline TOMM ${ }^{d}$ & 0 & 0 & & 2 & 1 & & 2 & 2 & \\
\hline $\mathrm{TIMM}^{\mathrm{e}}$ & 1 & 1 & & 0 & 0 & & 9 & 0 & 0.0029 \\
\hline Mitochondrial transcription & 2 & 0 & & 0 & 0 & & 5 & 1 & 0.0938 \\
\hline Mitochondrial ribosome, translational, processing, peptidase & 4 & 0 & 0.0625 & 0 & 0 & & 6 & 2 & 0.1093 \\
\hline
\end{tabular}

${ }^{a}$ CDNA microarray - Kong et al. (2011).

bShotgun proteomics-Kong B. et al. (2016).

${ }^{c}$ RNAseq-Bottje et al. (2017).

${ }^{*}$ Binomial (exact) $P$ values for $H$ vs. $L$ in each dataset.

${ }^{d}$ Transport protein outer mitochondrial membrane.

${ }^{e}$ Transport protein inner mitochondrial membrane.

TABLE 4 | Numbers of genes and proteins associated with transcription, pre-mRNA processing, and ribosomal assembly that were higher (H) or lower (L) in breast muscle of high feed efficiency (FE) compared to low FE pedigree broiler male for the terms provided obtained from global expression microarray, proteomics, and RNAseq datasets and for numbers of all three global expression datasets combined.

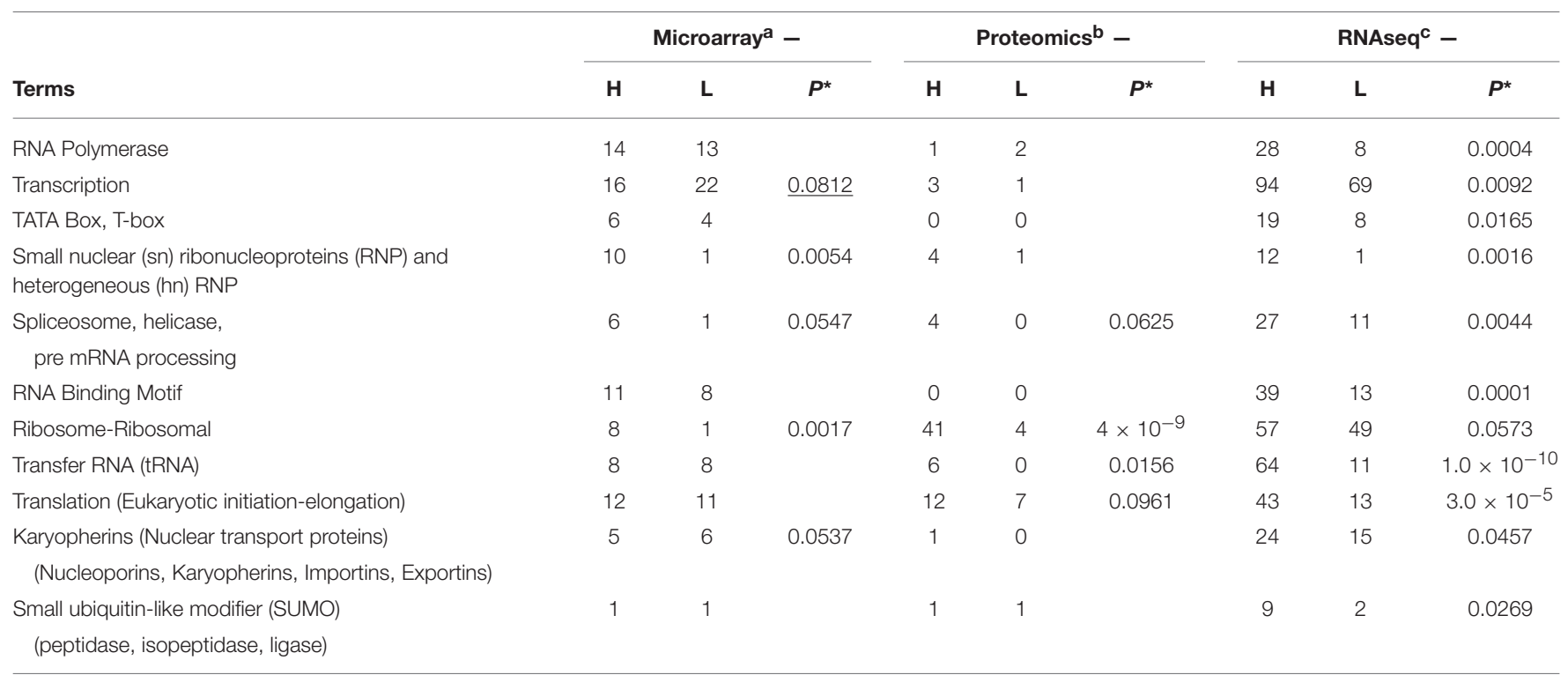

${ }^{a}$ CDNA microarray-Kong et al. (2011).

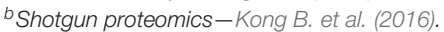

${ }^{c}$ RNAseq-Bottje et al. (2017).

*Binomial (exact) $P$ values for $H$ vs. $L$ (higher vs. lower) in each dataset.

$P$ values not underlined indicate a significant bionomial skew in high FE numbers.

$P$ values that are underlined indicate a significant skew in low FE numbers.

$P$ values that are blank are indicative of values $>0.10$.

Extraction of mRNA is a largely unbiased procedure that occurs with equal efficiency irrespective of the function of the encoded proteins. For all intents and purposes, every mRNA in a given tissue sample will be detected by modern screening approaches. On the other hand, proteomic extractions are very sensitive to the biochemical properties of the individual proteins, such as solubility, which can differ markedly both within and between organelles in general, and especially with the mitoproteome that contains a large number of proteins with hydrophobic regions, such as those associated with the electron transport chain (Lescuyer et al., 2003). In addition, proteomic analyses tend to be biased toward abundant structural proteins and may fail to identify less abundant but very important regulatory proteins such as transcription 
TABLE 5 | Numbers of genes and proteins associated with cytoskeletal-muscle fibers, phosphorylation regulation, cellular quality control mechanisms (proteosome and autophagy) that were higher (H) or lower (L) in breast muscle of high feed efficiency (FE) compared to low FE pedigree broiler male for the terms provided obtained from global expression microarray, proteomics, and RNAseq datasets and for numbers of all three global expression datasets combined.

\begin{tabular}{|c|c|c|c|c|c|c|c|c|c|c|}
\hline \multirow[b]{2}{*}{ Term } & \multicolumn{2}{|c|}{ Microarray $^{a}$} & \multirow[b]{2}{*}{$P^{*}$} & \multicolumn{3}{|c|}{ Proteomics $^{b}$} & \multicolumn{2}{|c|}{ RNAseq ${ }^{c}$} & \multirow[b]{2}{*}{$P^{*}$} & \multirow[b]{2}{*}{$\mathbf{H}$} \\
\hline & $\mathbf{H}$ & $\mathbf{L}$ & & $\mathbf{H}$ & $\mathbf{L}$ & $P^{*}$ & $\mathbf{H}$ & $\mathbf{L}$ & & \\
\hline \multicolumn{11}{|l|}{ PHOSPHORYLATION } \\
\hline Cytoskeletal-muscle fibers ${ }^{d}$ & 9 & 20 & $\underline{0.0004}$ & 7 & 21 & $\underline{0.0044}$ & 21 & 42 & $\underline{0.0030}$ & 35 \\
\hline Protein tyrosine phosphatase & 2 & 1 & & 2 & 0 & & 5 & 27 & $\underline{0.0020}$ & 9 \\
\hline Protein phosphatase & 4 & 2 & & 7 & 1 & 0.0313 & 30 & 24 & 0.0778 & 41 \\
\hline Tyrosine kinase & 6 & 4 & & 1 & 0 & & 11 & 31 & $\underline{0.001}$ & 17 \\
\hline \multicolumn{11}{|l|}{ QUALITY CONTROL } \\
\hline Proteosome & 7 & 3 & 0.1172 & 21 & 4 & 0.0004 & 31 & 4 & 2E-06 & 59 \\
\hline Autophagy & 6 & 2 & 0.1093 & 4 & 0 & 0.0625 & 36 & 15 & 0.0014 & 49 \\
\hline
\end{tabular}

${ }^{a}$ CDNA microarray - Kong et al. (2011).

${ }^{b}$ Shotgun proteomics-Kong B. et al. (2016).

${ }^{c}$ RNAseq-Bottje et al. (2017).

${ }^{a}$ Actin (ACTA), myosin (MYH), troponin (TNNT).

${ }^{*}$ Binomial (exact) $P$ values for $H$ vs. $L$ in each dataset. Blanks are indicative of $P>0.10$

$P$ values not underlined indicate a significant bionomial skew in high FE.

$P$ values that are underlined indicate a significant skew in low FE.

$P$ values that are blank are indicative of values $>0.10$.

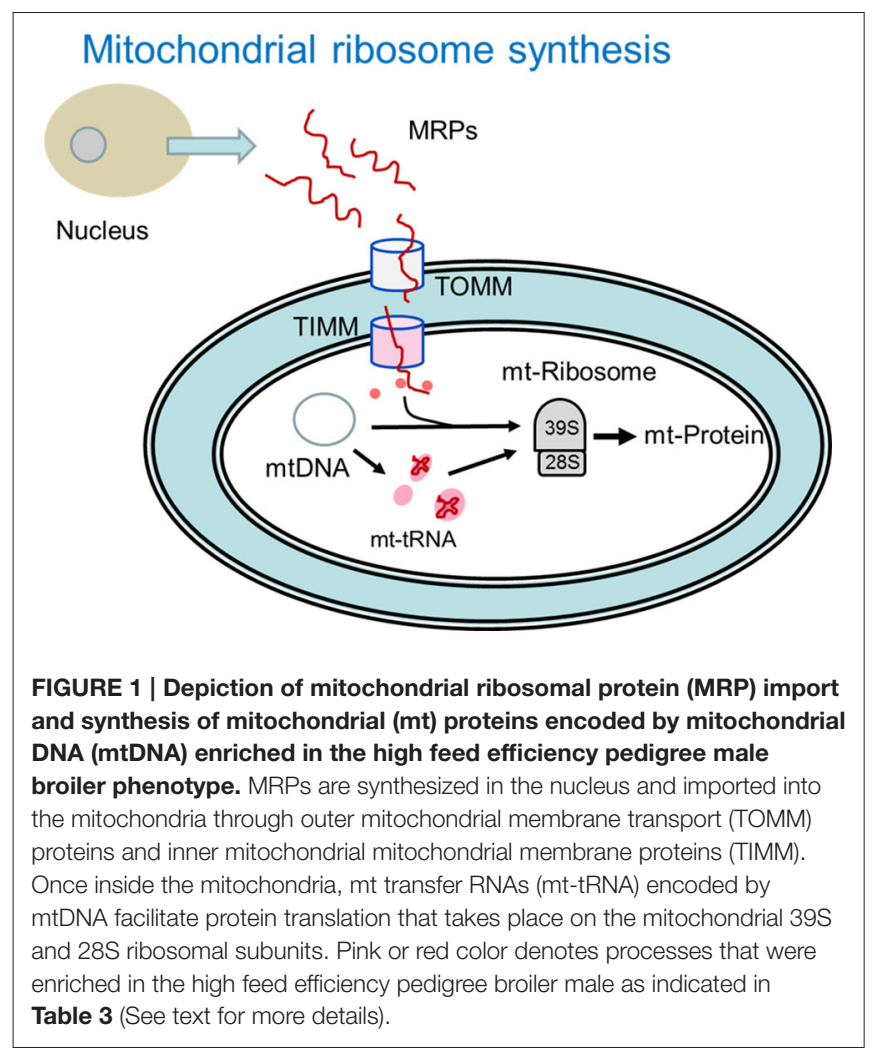

factors. In our case, the RNAseq and microarray analyses detected 103 and 30 transcripts encoding mitochondrial proteins, respectively, compared to 5 mitochondrial proteins for the terms shown in Table 3. This discrepancy is also related to the total numbers of molecules that were detected; $\sim 1,800$ proteins compared to more than 4,000 and 10,000 gene transcripts in the microarray and RNAseq datasets, respectively.

\section{Ribosome Assembly Machinery, Nuclear Transport, and Protein Translation Enrichment in High FE Muscle}

Over 1,500 mitochondrial proteins are encoded by nuclear $(n)$ DNA and imported into the mitochondria, to form enzyme complexes of the electron transport chain, Kreb cycle, and other mitochondrial processes (Wallace, 1999; Sue and Schon, 2000; Lescuyer et al., 2003; Taylor et al., 2003; Chevallet et al., 2006). Thus, mitoproteome expression is highly dependent on nuclear transcription and protein translation taking place on cytoplasmic ribosomes. Based on a review by Wahl et al. (2009), the global expression datasets were searched for terms associated with RNA synthesis, ribosome assembly, protein translating activity, small nuclear (sn) ribonucleoproteins (RNPs) and heterogenous (hn) SNPs, and expression of nuclear pore molecules including nucleoporins, karyopherins, importins, and exportins (Table 4). Transcription of ribosomal $(\mathrm{r})$, messenger $(\mathrm{m})$, and transfer $(\mathrm{t})$ RNAs is initiated by RNA polymerases I, II, and III (Roeder and Rutter, 1969, 1970; Sentenac, 1985). Although, there was no difference in the microarray and proteomic data, RNA polymerase was enriched in the high FE phenotype in the RNAseq data (Table 4). Binomial analysis of the combined data, however, revealed significant skew of RNA polymerase toward the high FE phenotype (data not shown). The findings suggest that RNA polymerase was enriched in the high FE phenotype but further study is needed to determine if this difference would translate to higher RNA polymerase activity in the high FE phenotype. 
When "transcription" was used as the search term, binomial distribution analysis revealed a marginal enrichment in the low FE phenotype enrichment $(P=0.08)$ in the microarray data but a significant enrichment in the high FE phenotype with the RNAseq dataset (Table 4). The reason for the discrepancy in the two transcript analyses is not apparent at this time. Typically, transcription promotors contain a sequence that recognizes the promotor nucleotide base sequences (TATA), and recruits TATAbinding proteins (TBP) to the site on DNA where transcription begins following RNA polymerase activity (Cormack and Struhl, 1992; Schultz et al., 1992; Pugh, 2000; Davidson, 2003). The TATA box (and T-box) is considered to be the core promoter sequence and the binding site of general transcription factors and histones; the binding of a transcription factor blocks the binding of a histone and vice versa. Higher numbers of genes associated with TATA box and T-box may serve to facilitate gene transcription in the high FE phenotype (Table 4).

The removal of introns in the nucleus is accomplished by coordinated activities involving proteins and RNAs found in the spliceosome (see Wahl et al., 2009). In this premRNA processing step, and during transcription of mRNA and tRNA prior to export from the nucleolus and nucleus, are numerous RNA binding proteins targeting RNA binding motifs. Spliceosome, helicase, and pre-mRNA processing components were all enriched in the high FE phenotype (Table 4).

As protein synthesis strongly correlates with cellular rRNA and tRNA content, cells need to sustain high rates of RNA polymerase I and III transcription to synthesize as many as 2 million ribosomes per cell (Drygin et al., 2010). Ribosomes and tRNA enrichment was observed in the high FE phenotype (Table 4). Although, no mitochondrial ribosomal proteins were detected with shotgun proteomics (Table 3), many ribosome and ribosomal-related proteins were detected and were enriched in the high FE phenotype (Table 4). Protein translation is facilitated by eukaryotic initiation and elongation proteins. While there was no difference in the numbers of initiation and elongation protein transcripts in the microarray dataset, marginal and significant enrichments were observed in the proteomics and RNAseq datasets, respectively (Table 4). Collectively, these results indicate that requisite architecture for transcription and translation processes were enriched in breast muscle of the high FE compared to the low FE PedM broiler.

The nucleopore complex is vital in separating the nucleoplasm from the cytoplasm, and in trafficking of RNA components (e.g., mRNAs, tRNAs, and rRNAs) out of the nucleus, and cytoplasmic synthesized proteins into the nucleus that are requisite for RNA synthesis outlined above. The nucleopore complex is comprised of a large family of karyopherin proteins with several protein subfamilies; e.g., nucleoporins, importins, and exportins (see review by Wente and Rout, 2010). The action of these proteins in nuclear transport are modulated by small ubiquitin-like modifying (SUMO) proteins that are in turn regulated by SUMO peptidase and ligase activities. The high FE phenotype exhibited a significant enrichment of nuclear transport proteins as well as with SUMO proteins for the RNAseq data analysis (Table 4) that could be hypothesized to favor nuclear transport and/or regulation of
RNA and protein transport between the nuclear and cytoplasm compartments.

\section{Enrichment of Cytoarchitecture-Muscle Fibers in Low FE Muscle}

The low numbers of proteins relative to the number of transcripts observed in the proteomic dataset in Tables $2-4$ could be due to fewer proteins being detected compared to transcriptomic analyses, as well as the technical limitations of protein detection as mentioned previously. In the microarray data, we reported a general down-regulation of many transcripts associated with cytoskeletal architecture and/or muscle fibers (Kong et al., 2011; Bottje and Kong, 2013) that was also observed in the RNAseq study (Bottje et al., 2017). The implications of these findings on muscle cytoarchitecture in the high and low FE groups were discussed in detail in Bottje et al. (2017) and won't be repeated here. A search for actin (ACTA), myosin (MYH) and troponin (TNNC) yielded results indicating enrichment of these molecules in the low FE muscle phenotype across all three global expression datasets (Table 5). The numbers of actin, myosin and troponin in the proteomics dataset were similar to numbers of transcripts in the microarray dataset and may reflect that abundances of these proteins were much greater (e.g., >100 spectral counts) compared to normalized spectral counts ranging from 5 to 30 for proteins listed in Tables 3, 4.

\section{Phosphorylation Regulation}

Activation or deactivation of enzymes and numerous cascade pathways are carried out by a large number of diverse phosphatases and kinases (e.g., see reviews: Hunter and Cooper, 1985; Hubbard and Till, 2000; Vishup, 2000; Mustelin, 2007). Although, we did not do an exhaustive search in these areas, a few are provided indicating that certain components of cell metabolism were enhanced in the low FE phenotype. Whereas protein tyrosine phosphatase and protein tyrosine kinase families were enriched in the low FE phenotype, protein phosphatase was enriched in the high FE phenotype (Table 5). Because of the diverse nature of phosphorylation reactions, it is very difficult to speculate the role that this apparent differential enrichment of phosphatases and kinases have in contributing to a high or low FE phenotype. Nonetheless, it is apparent that there are distinct differences and additional research is needed in order to fully characterize the role that individual enzymes might have in the phenotypic expression of feed efficiency.

\section{Enrichment of Quality Control Processes: Proteosome and Autophagy}

Interestingly, enrichment of protein and/or organelle degradation associated with cellular quality control mechanisms of proteosome and autophagy processes was observed in the high FE phenotype (Table 5). At the outset, these processes appear to be a counterproductive to cellular efficiency as protein degradation and resynthesis requires tremendous amounts of energy. Based on significant differences in gene expression, we had previously hypothesized that proteosomal activity was increased in the high FE phenotype (Bottje et al., 2014). This hypothesis is supported in the present study in which 
both proteomic and transcriptomic data indicate proteosomal enrichment in the high FE phenotype (Table 5). Possibly, enhanced proteosomal activity in the high $\mathrm{FE}$ muscle serves to recycle proteins at a more rapid rate, keeping the overall functionality of proteins optimal in high FE. Furthermore, the proteosome is not only involved in protein hydrolysis and protein resynthesis, it may also support a high degree of protein functionality through refolding when the tertiary structure becomes misaligned (Hershko and Ciechanover, 1986; Ciechanover, 1998; Lecker et al., 2006). An additional role of proteosomal activity has also been proposed as an important component of transcription (see review, Muratani and Tansey, 2003). In this model, the ubiquitin-proteosome complex interacts with general transcription machinery that includes ubiquitinylation of RNA polymerase II that in turn recruits the $26 \mathrm{~S}$ proteosome (composed of the $19 \mathrm{~S}$ and $20 \mathrm{~S}$ proteosome). The $26 \mathrm{~S}$ proteosome then functions to maintain optimal promoter-activator interactions between the RNA polymerase II-26S proteosome complex. Based on gene expression in the microarray dataset, we had proposed that formation of the RNA polymerase II pre-initiation complex would favor enhanced transcription processes in the high FE phenotype (Bottje et al., 2014). In vitro, the proteosome has been shown to bind to unfolded proteins, stimulate refolding, and release the protein back into the cytosol in its original tertiary/optimal structure form (Braun et al., 1999; Strickland et al., 2000; Glickman and Cienhanover, 2002). The proteosome has also been shown to inhibit the aggregation of misfolded proteins in the cytosol (Braun et al., 1999). Thus, the enrichment of proteosomes in the high FE could help maintain optimal functionality of proteins in the cell either by rapid degradation and resynthesis of damaged proteins or maintaining optimal protein tertiary structure. Interestingly, a new homeostatic role of mitochondria in the cell may be in complementing proteosome activity through degradation of protein aggregates formed in the cytosol that are imported into the mitochondria (Ruan et al., 2017). Thus, it is possible that the enhanced expression of the mitoproteome (Kong B. et al., 2016; Bottje et al., 2017) could also function in quality control of damaged components in the cell.

Autophagy (self-eating) is another degradative repair process that degrades damaged structures ranging from proteins to entire organelles (e.g., mitochondria, endoplasmic reticulum; Klionsky, 2005; Massey et al., 2006; Tasdemir et al., 2007; Levine and Kroemer, 2008). During starvation, autophagy can generate energy via protein and lipid catabolism and remove damaged components followed by recycling of amino acids for new de novo synthesis of proteins (e.g., Hamasaki et al., 2008). Although, this system has been well characterized in organisms ranging from yeast to mammals, the first characterization of autophagy expression in avian tissues and cells was only reported recently (Piekarski et al., 2014). Evidence that components of autophagy pathway were up-regulated in the high FE phenotype (from the same tissues that were used in the global gene and protein expression datasets in the current study) has been reported (Piekarski, 2015).

\section{Summary}

In this study, we have taken an approach in investigating global gene and protein expression datasets to answer essentially "yes-no" questions; For a given cellular process (e.g., ribosome assembly), are there greater numbers of genes or proteins expressed at higher levels (enriched) in the high or low FE phenotype? This analysis builds upon a series of studies that have been conducted with this line of PedM broilers individually phenotyped for FE (reviewed in Bottje and Carstens, 2009; Bottje and Kong, 2013). These studies pointed toward mitochondria playing an important role in FE in this line of birds on several fronts: (1) higher electron transport chain coupling and less proton leak in high FE mitochondria, and (2) increased reactive oxygen species production in low FE mitochondria. With evidence that the mitoproteome was enriched in the high FE phenotype (Kong B. et al., 2016; Bottje et al., 2017), we investigated mechanisms associated with mitochondrial and cytosolic ribosomal assembly determined that both were enriched in breast muscle of the high FE compared to the low FE PedM broiler phenotype (Figure 1, Tables 2, 3). Since the mitoproteome is comprised mainly of nDNA encoded proteins, full mitoproteome expression depends on a large contingent of cytosolic translated proteins. The requisite architecture for cytosolic ribosomal assembly and translation was also enriched in the high FE phenotype (Table 4, Figure 2). It should be noted that the expression of many enzymes in nucleic and ribonucleic acid synthesis, including rate limiting steps, were significantly elevated in the high FE phenotype (Bottje and Kong, 2017). Additionally, enrichment of nuclear transport proteins responsible for maintaining separate nuclear and cytosolic compartments and regulating movement of molecules in and out of the nucleus were enriched in the high FE phenotype (Table 4, Figure 2). Enrichment of cytoskeletal/muscle fibers was observed in the low FE phenotype that we have previously hypothesized could require increased energy expenditure in low FE and contribute to the phenotypic expression of low FE (Kong et al., 2011). Differences in enrichment of phosphorylation and kinase families were also observed between the high and low FE phenotypes (Table 5). Finally, quality control processes carried out by proteosomes and autophagy were enriched in the high FE phenotype leading us to speculate that cellular efficiency may involve energy expenditure for degradation and resynthesis in order to maintain optimal protein and organelle function (Table 5). We have also observed up-regulation of mitochondrial adenine nucleotide translocase and voltage dependent activated channel that would facilitate phosphate transfer out of the mitochondria to replenish ATP via the creatine-phosphocreatine shuttle system in the high FE phenotype (Bottje et al., 2017). This creatinephosphocreatine shuttle could presumably provide a means of continual energy supply needed for resynthesis of proteins and organelles degraded by the proteosome and autophagy systems; the payback to the cell being maintenance of optimal overall cell function.

It is apparent that feed efficiency is a very complex genetic trait. Development of an accurate biomarker, or biomarker panel 


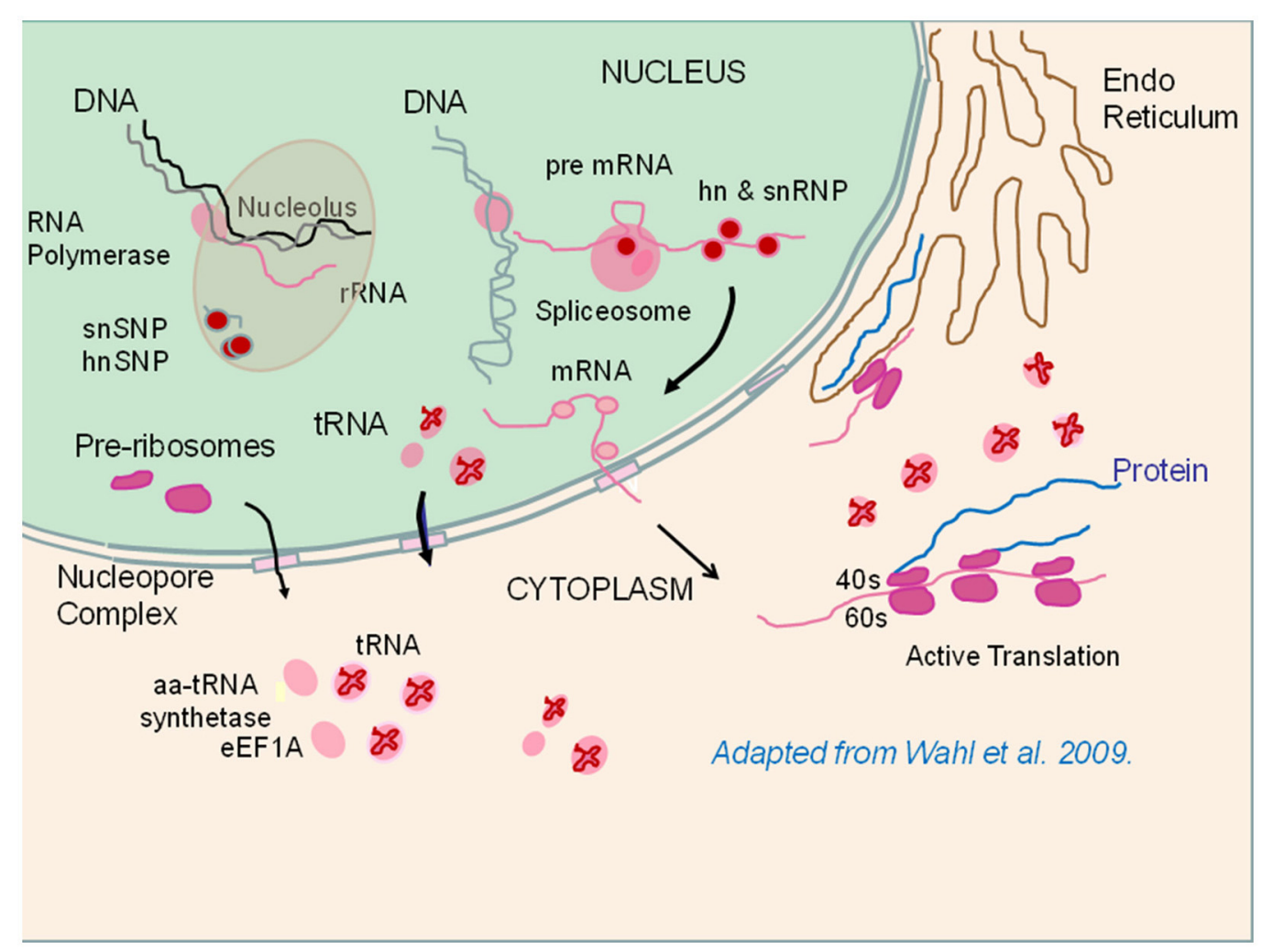

FIGURE 2 | Depiction of ribosome assembly and protein translation processes that were enriched in the high feed efficiency (FE) pedigree male broiler phenotype. Components shown in pink or red were enriched in the high FE phenotype (see Table 4). RNA polymerases initiate transcription in the nucleoplasm or in the nucleolus. Small nuclear (sn) ribonucleoproteins (SNP) and heterogeneous (hn) SNP play roles in nucleolar ribosomal RNA (rRNA) synthesis as well as in messenger RNA (mRNA) synthesis in conjunction with spliceosome activity responsible for cleaving on introns from the pre mRNA molecule. Pre-ribosomes as well as tRNAs and mRNAs are exported out of the nucleus into the cytoplasm. In the cytoplasm, ribosomes assemble into the $40 \mathrm{~S}$ and $60 \mathrm{~S}$ subunits where protein translation takes place in conjunction with delivery of amino acids by tRNA (See text for details). The figure is adapted from Figure 1 (p. 702) of Wahl et al. (2009).

would be very desirable to aid genetic selection programs. As difficult and elusive as this may be, we hope that this study will add to overall understanding of feed efficiency at the cellular level and will help point researchers in the right directionwhatever that might be. In this report we feel we have provided a convincing argument that there is a functional genomic architecture that would foster transcription and translation processes in the high FE phenotype. Trying to fit a biomarker tool onto this area of cellular metabolism will be difficult due to the inherent complexity. Nevertheless, when effective tools of biomarker selection are developed and implemented, it will help the world in meeting the increasing demands for animal protein that that are with us now, and will only be greater in the future.

\section{AUTHOR CONTRIBUTIONS}

$\mathrm{WB}, \mathrm{BK}$, and $\mathrm{NH}$ conceived, designed, and conducted the experiments. Data analysis was conducted by $\mathrm{WB}, \mathrm{BK}, \mathrm{NH}$, and
AR. The paper was written through contributions and critical review of the manuscript by all authors (WB, KL, AP, SD, AR, $\mathrm{NH}$, and $\mathrm{BK})$.

\section{FUNDING}

This work is supported by USDA-NIFA (\#2013-01953), Arkansas Biosciences Institute (Little Rock, AR) and the Agricultural Experiment Station (University of Arkansas).

\section{ACKNOWLEDGMENTS}

With the diverse areas mentioned in this study, we recognize that in many cases citations of review papers have been made at the expense of the original research upon which the review papers were written. For this reason, we would like to apologize to researchers that were not referenced here. I (WB) would like to dedicate this manuscript to my mother, Joyce Bottje, who gave me the spirit of adventure. 


\section{REFERENCES}

Arthur, P. F., and Herd, R. M. (2005). Efficiency of feed utilisation by livestockImplications and benefits of genetic improvement. Can. J. Anim. Sci. 85, 281-290. doi: 10.4141/A04-062

Avadhani, N. G., and Buetow, D. E. (1974). Mitochondrial and cytoplasmic ribosomes: distinguishing characteristics and a requirement for the homologous ribosomal salt-extractable fraction for protein synthesis. Biochem. J. 140, 73-78. doi: 10.1042/bj1400073

Bottje, W., and Carstens, G. E. (2009). Association of mitochondrial function and feed efficiency in poultry and livestock species. J. Anim. Sci. 87, E48-E63. doi: $10.2527 /$ jas.2008-1379

Bottje, W. G., Kong, B.-W., Lee, J. Y., Washington, T., Baum, J. I., Dridi, S., et al. (2014). Potential roles of mTOR and protein degradation pathways in the phenotypic expression of feed efficiency in broilers. J. Biochem. Physiol. 3:125. doi: 10.4172/2168-9652.1000125

Bottje, W. G., Kong, B., Reverter, A., Waardenberg, A. J., Lassiter, K., and Hudson, N. J. (2017). Progesterone signalling in broiler skeletal muscle is associated with divergent feed efficiency. BMC Syst. Biol. 11:29. doi: 10.1186/s12918-017-0396-2

Bottje, W. G., and Kong, B.-W. (2017). "Cellular basis of feed efficiency in muscle: focus on mitochondria and nucleic acid metabolism," in Achieving Sustainable Production of Poultry Meat, ed T. Appelgate (Sawston Cambridge: Burleigh Dodds Science Publishing Ltd.), 1-6.

Bottje, W. G., Kong, B.-W., Song, J. J., Lee, J. Y., Hargis, B. M., Lassiter, K., et al. (2012). Gene expression in breast muscle associated feed efficiency in a single male broiler line using a chicken $44 \mathrm{k}$ microarray. II. Differentially expressed focus genes. Poultry Sci. 91, 2576-2587. doi: 10.3382/ps.201202204

Bottje, W., Iqbal, M., Tang, Z. X., Cawthon, D., Okimoto, R., Wing, T., et al. (2002). Association of mitochondrial function with feed efficiency within a single genetic line of male broilers. Poult. Sci. 81, 546-555. doi: 10.1093/ps/81. 4.546

Bottje, W., and Kong, B. W. (2013). Feed efficiency: mitochondrial function to global gene expression. J. Anim. Sci. 91, 1582-1593. doi: 10.2527/jas.2012-5787

Brand, M. D. (1990). The contribution of the leak of protons across the mitochondrial inner membrane to standard metabolic rate. J. Theor. Biol. 145, 267-286. doi: 10.1016/S0022-5193(05)80131-6

Braun, B. C., Glickman, M. H., Kraft, R., Dahlmann, B., Kloetzel, P. M., Finely, D., et al. (1999). The base of the proteasome regulatory particle exhibits chaperone-like activity. Nat. Cell Biol. 1, 221-226. doi: 10.1038/12043

Chevallet, M., Lescuyer, P., Diemer, H., Dorsselaer, A. V., Wagner-Leize, E., and Rabbiloud, T. (2006). Alterations of the mitochondrial proteome caused by the absence of mitochondrial DNA: a proteomic view. Electrophoresis 27, 1574-1583. doi: 10.1002/elps.200500704

Ciechanover, A. (1998). The ubiquitin-proteasome pathway: on protein death and cell life. EMBO J. 17, 7151-7160. doi: 10.1093/emboj/17.24.7151

Cormack, B. P., and Struhl, K. (1992). The TATA-binding protein is required for transcription by all three nuclear RNA polymerases. Cell 69, 685-696. doi: 10.1016/0092-8674(92)90232-2

Davidson, I. (2003). The genetics of TBP and TBP-related proteins. Trends Biochem. Sci. 28, 391-398. doi: 10.1016/S0968-0004(03)00117-8

Drygin, D., Rice, G. R., and Grummt, I. (2010). The RNA polymerase transcription machinery: an emerging target for the treatment of cancer. Ann. Rev. Pharmacol. Toxicol. 50, 131-156. doi: 10.1146/annurev.pharmtox.010909.105844

Fox, T. D. (2012). Mitochondrial protein synthesis, import, and assembly. Genetics 192, 1203-1234. doi: 10.1534/genetics.112.141267

Glickman, M. H., and Cienhanover, A. (2002). The ubiquitin-proteosome proteolytic pathway: destruction for the sake of construction. Physiol. Rev. 82, 373-428. doi: 10.1152/physrev.00027.2001

Graack, H., Bryant, M. L., and O’Brien, T. W. (1999). Identification of mammalian ribosomal proteins (MRPs) by $\mathrm{N}$-terminal sequencing of purified bovine MRPs and comparison to databank sequences: the large subribosomal particle. Biochemistry 38, 16569-16577. doi: 10.1021/bi991543s

Hamasaki, M., Furuta, N., Matsuda, A., Nezu, A., Yamamoto, A., Fujita, N., et al. (2008). Autophagosomes form at ER-mitochondria contact sites. Nature 495, 389-393. doi: 10.1038/nature11910
Hershko, A., and Ciechanover, A. (1986). The ubiquitin pathway for the degradation of intracellular proteins. Prog. Nucleic Acid Res. Mol. Biol. 33, 19-56. doi: 10.1016/S0079-6603(08)60019-7

Hubbard, S. R., and Till, J. H. (2000). Protein tyrosine kinase structure and function. Ann. Rev. Biochem. 69, 373-398. doi: 10.1146/annurev.biochem.69.1.373

Hudson, N. J., Darylmple, B. P., and Reverter, A. (2012). Beyond differential expression: the quest for causal mutations and effector molecules. BMC Genomics 13:356. doi: 10.1186/1471-2164-13-356

Hudson, N. J., Reverter, A., and Dalrymple, B. P. (2009). A differential wiring analysis of expression data correctly identifies the gene containing the causal mutation. PLoS Comp. Biol. 5:e1000382. doi: 10.1371/journal.pcbi.1000382

Hunter, T., and Cooper, J. A. (1985). Protein-tyrosine kinase. Ann. Rev. Biochem. 54, 897-930. doi: 10.1146/annurev.bi.54.070185.004341

Jolly, R. A., Goldstein, K. M., Wei, T., Gao, H., Chen, P., Huang, S., et al. (2005). Pooling samples within microarray studies: a comparative analysis of rat liver transcription response to prototypical toxicants. Physiol. Genomics 22, 346-335. doi: 10.1152/physiolgenomics.00260.2004

Kendziorski, C., Irizarry, R. A., Chen, K.-S., Haag, J. D., and Gould, M. N. (2005). On the utility of pooling biological samples in microarray experiments. Proc. Natl. Acad. Sci. U.S.A. 102, 4252-4257. doi: 10.1073/pnas.05006 07102

Klionsky, D. J. (2005). The molecular machinery of autophagy: unanswered questions. J. Cell Sci. 118, 7-18. doi: 10.1242/jcs.01620

Kong, B., Lassiter, K., Piekarski-Welsher, A., Dridi, S., Reverter-Gomez, A., Hudson, N. J., et al. (2016). Proteomics of breast muscle tissue associated with the phenotypic expression of feed efficiency within a pedigree male broiler line: I. Highlight on mitochondria. PLOS ONE 11:e0155679. doi: 10.1371/journal.pone.0155679

Kong, B., Song, J., Lee, J. Y., Hargis, B. M., Wing, T., Lassiter, K., et al. (2011). Gene expression in breast muscle associated feed efficiency in a single male broiler line using a chicken $44 \mathrm{k}$ microarray. I. Top differentially expressed genes. Poult. Sci. 90, 2535-2547. doi: 10.3382/ps.2011-01435

Kong, R. S., Liang, G., Chen, Y., Stothand, P., and Guan, L. L. (2016). Transcriptome profiling of the rumen epithelium of beef cattle differing in residual feed intake. BMC Genomics 17:502. doi: 10.1186/s12864-016-2935-4

Lecker, S. H., Goldberg, A. L., and Mitch, W. E. (2006). Protein degradation by the ubiquitin-proteasome pathway in normal and disease states. J. Am. Soc. Nephrol. 17, 1807-1819. doi: 10.1681/ASN.2006010083

Lescuyer, P., Strub, J., Luche, S., Diemer, H., Martinez, P., Dorsselaer, A. V., et al. (2003). Progress in the definition of a reference human mitochondrial proteome. Proteomics 3, 157-167. doi: 10.1002/pmic.200390024

Levine, B., and Kroemer, G. (2008). Autophagy in the pathogenesis of disease. Cell 132, 27-42. doi: 10.1016/j.cell.2007.12.018

Massey, A. C., Zhang, C., and Cuervo, A. M. (2006). Chaperone-mediated autophagy in aging and disease. Curr. Top. Dev. Biol. 73, 205-235. doi: 10.1016/S0070-2153(05)73007-6

Muratani, M., and Tansey, W. P. (2003). How the ubiquitin-proteosome systems controls transcription. Nat. Rev. 4, 1-10. doi: 10.1038/nrm1049

Mustelin, T. (2007). A brief introduction to the protein phosphatase families. Meth Mol. Biol. 365, 9-22. doi: 10.1385/1-59745-267-X:9

O'Brien, T. W. (2002). Evolution of a protein-rich mitochondrial ribosome: implications for human genetic disease. Gene 286, 73-79. doi: 10.1016/S0378-1119(01)00808-3

Piekarski, A. (2015). Autophagy and Its Potential Role in Stress and Feed Efficiency in Avian Tissues. University of Arkansas, (Fayetteville, AR).

Piekarski, A., Khaldi, S., Greene, E., Lassiter, K., Mason, J. G., Anthony, N., et al. (2014). Tissue distribution, gender- and genotype-dependent expression of autophagy-related genes in avian species. PLOS ONE 9:e112449. doi: 10.1371/journal.pone.0112449

Pugh, B. F. (2000). Control of gene expression through regulation of the TATbinding protein. Gene 255, 1-14. doi: 10.1016/S0378-1119(00)00288-2

Roeder, R. G., and Rutter, W. G. (1970). Specific nucleolar and nucleoplasmic RNA polymerases. Proc. Natl. Acad. Sci. U.S.A. 65, 675-682. doi: 10.1073/pnas.65.3.675

Roeder, R. G., and Rutter, W. J. (1969). Multiple forms of DNA-dependent RNA polymerase in eukaryotic organisms. Nature 224, 234-237. doi: $10.1038 / 224234 \mathrm{a} 0$ 
Rolfe, D. F., and Brand, M. D. (1996). Contribution of mitochondrial proton leak to skeletal muscle respiration and to standard metabolic rate. Am. J. Physiol. 271, 1380-1389.

Rolfe, D. F., and Brand, M. D. (1997). The physiological significance of mitochondrial proton leak in animal cells and tissues. Biosci. Rep. 17, 9-16. doi: 10.1023/A:1027327015957

Rolfe, D. F. S., Newman, J. M., Buckingham, J. A., Clark, M. G., and Brand, M. D. (1999). Contribution of mitochondrial proton leak to respiration rate in working skeletal muscle and liver and to SMR. Am. J. Physiol. 276, C692-C699.

Ruan, L., Zhou, C., Jin, E., Kucharavy, A., Zhang, W., Florens, L., et al. (2017). Cytsolic proteostasis through importing of misfolded proteins into mitochondria. Nature 543, 443-446. doi: 10.1038/nature21695

Scheiber, G. L., and O'Brien, T. W. (1985). Site of synthesis of the proteins of mammalian mitochondrial ribosomes under nondenaturing conditions. J. Biol. Chem. 260, 6367-6372.

Schultz, M. C., Reeder, R. H., and Hahn, S. (1992). Variants of the TATA-binding protein can distinguish subsets of RNA polymerase I, II, and II promoters. Cell 69, 697-702. doi: 10.1016/0092-8674(92)90233-3

Sentenac, A. (1985). Eukaryotic RNA polymerases. CRC Crit. Rev. Biochem. 18, 31-90. doi: 10.3109/10409238509082539

Strickland, E., Hakala, K., Thomas, P. J., and Demartino, G. N. (2000). Recognition of misfolding proteins by PA700, the regulatory subcomplex of the $26 \mathrm{~S}$ proteasome. J. Biol. Chem. 275, 5565-5572. doi: 10.1074/jbc.275.8.5565

Sue, C. M., and Schon, E. A. (2000). Mitochondrial chain diseases and mutations in nuclear DNA: a promising start. Brain Pathol. 10, 442-450. doi: 10.1111/j.1750-3639.2000.tb00276.x

Sylvester, J. E., Fischel-Ghodsian, N., Mougey, E. B., and O'Brien, T. W. (2004). Mitochondrial ribosomal proteins: candidate genes for mitochondrial disease. Genet. Med. 6, 73-80. doi: 10.1097/01.GIM.0000117333.21213.17

Tasdemir, E., Miuri, M. C., Tajaeddine, N., Criollo, A., Vicencio, M., Hickman, J. A., et al. (2007). Cell cycle-dependent induction of autophagy, mitophagy, and reticulophagy. Cell Cycle 6, 2263-2267. doi: 10.4161/cc.6.18.4681
Taylor, S. W., Fahy, E., Zhang, B., Glenn, G. M., Warnock, D. E., Wiley, S., et al. (2003). Characterization of the human heart mitochondrial proteome. Nat. Biotechnol. 12, 281-286. doi: 10.1038/nbt793

Vishup, D. M. (2000). Protein phosphatase 2A: a panoply of enzymes. Curr. Opin. Cell Biol. 12, 180-185. doi: 10.1016/S0955-0674(99)0 0074-5

Wahl, M. D., Will, C. L., and Luhrmann, R. (2009). The spliceosome: design principles of a dynamic RNP machine. Cell 136, 701-718. doi: 10.1016/j.cell.2009.02.009

Wallace, D. (1999). Mitochondrial diseases in man and mouse. Science 283, 1482-1488. doi: 10.1126/science.283.5407.1482

Wente, S. R., and Rout, M. P. (2010). The nuclear pore complex and nuclear transport. Cold Springs Harb. Perspect. Biol. 1, 1-14. doi: 10.1101/cshperspect.a000562

Zurlo, F., Larson, K., Bogardus, C., and Ravussin, E. (1990). Skeletal muscle metabolism is a major determinant of resting energy expenditure. J. Clin. Invest. 86, 1423-1427. doi: 10.1172/JCI114857

Conflict of Interest Statement: The authors declare that the research was conducted in the absence of any commercial or financial relationships that could be construed as a potential conflict of interest.

The reviewer DC and handling Editor declared their shared affiliation, and the handling Editor states that the process nevertheless met the standards of a fair and objective review.

Copyright (c) 2017 Bottje, Lassiter, Piekarski-Welsher, Dridi, Reverter, Hudson and Kong. This is an open-access article distributed under the terms of the Creative Commons Attribution License (CC BY). The use, distribution or reproduction in other forums is permitted, provided the original author(s) or licensor are credited and that the original publication in this journal is cited, in accordance with accepted academic practice. No use, distribution or reproduction is permitted which does not comply with these terms. 